\title{
Recent results from CODALEMA and the Nançay radio facilities related to cosmic-ray measurements
}

\author{
Richard Dallier ${ }^{1,2, \star}$, Didier Charrier ${ }^{1,2}$, Laurent Denis ${ }^{2}$, Antony Escudie ${ }^{1}$, Florian Gaté ${ }^{1}$, Alain \\ Lecacheux $^{3}$, Lilian Martin ${ }^{1,2}$, and Benoît Revenu ${ }^{1,2}$ \\ ${ }^{1}$ SUBATECH - Ecole des Mines de Nantes - CNRS/IN2P3 - Université de Nantes, 4 rue Alfred Kastler, 44307 \\ Nantes cedex 3, France \\ ${ }^{2}$ U.S. Nançay - Observatoire de Paris, CNRS/INSU, 18330 Nançay, France \\ ${ }^{3}$ LESIA - Observatoire de Paris, CNRS/INSU, 5 place Jules Janssen, 92190 Meudon, France
}

\begin{abstract}
Since 2003, the Nançay Radio Observatory hosts the CODALEMA experiment, dedicated to radio detection of cosmic ray induced extensive air showers. CODALEMA also features the R\&D EXTASIS project, aiming at detecting the lowfrequency signal ([2-6] $\mathrm{MHz}$ ) produced at the sudden disappearance of the air shower particles hitting the ground. The 3 current antenna arrays present different antenna density and extent, and can be operated in a joint mode to record simultaneously the radio signal coming from air showers. Therefore, the Nançay facilities may offer a complete description of the air shower induced electric field at small, medium and large scale, and over an unique and very wide frequency band (from $\sim 2$ to $200 \mathrm{MHz}$ ).
\end{abstract}

\section{Radio detection: performances and limitations}

The radio technique is now used in a flurry of experiments throughout the world, among which AERA [1] and LOFAR [2] are the best known. These experiments present different bandwidth and detector densities. AERA measures showers in [30-80] MHz; LOFAR uses either a low frequency ([3080] $\mathrm{MHz}$ ) or a high frequency window ([110-190] MHz) (not simultaneously). On AERA, the detection technique is tested over $\sim 17 \mathrm{~km}^{2}$, with a sparse array of autonomous radio detector stations, similar in principle to the ones hereafter described for CODALEMA-3. Contrariwise, LOFAR features a very dense core of radio antennas, externally triggered by a particle detector, over an area of $\sim 0.2 \mathrm{~km}^{2}$ allowing one to study the electric field profile of individual air showers at small distances. But either triggered by ground particle arrays or in standalone mode, radio detectors achieving EAS detection still exhibit strong internal or external limitations (parasitic radio transients, limitations on detection of highly inclined EAS for externally triggered arrays, low detection range even in the [3080] $\mathrm{MHz}$ band, obvious contradiction between the high antenna density required for an optimal $X_{\max }$ reconstruction and the need to cover large areas...). Overcoming those limitations would require new instrumental developments, that are difficult to implement on currently running experiments without strong modifications. Therefore the CODALEMA-3/EXTASIS facilities at the Nançay radio observatory aim to combine a very wide bandwidth (from $2 \mathrm{MHz}$ up to $200 \mathrm{MHz}$ ), variable antenna density

\footnotetext{
^e-mail: richard.dallier@ subatech.in2p3.fr
} 
and smart triggering algorithms to explore some possible solutions to each of the above problems. In this paper, we will shortly describe the Nançay experimental site facilities devoted to radio detection of UHECR, and we present some performances recently reached with these multi-scale and multi-frequency facilities.

\section{The Nançay facilities and the CODALEMA-3/EXTASIS experiments}

Nowadays, the CODALEMA-3/EXTASIS experiment is composed of 5 arrays of detectors. The most ancient, installed for CODALEMA-2, is the particle detector array, while CODALEMA-3 was developed later with standalone radio detectors and two new cabled arrays of antennas (the so-called "compact array" and the composite trigger cluster) aiming at building a purely radio trigger. At last, a sparse array of 7 low-frequency antennas has been installed for the EXTASIS project. Figure 1 shows the map of the current setups.

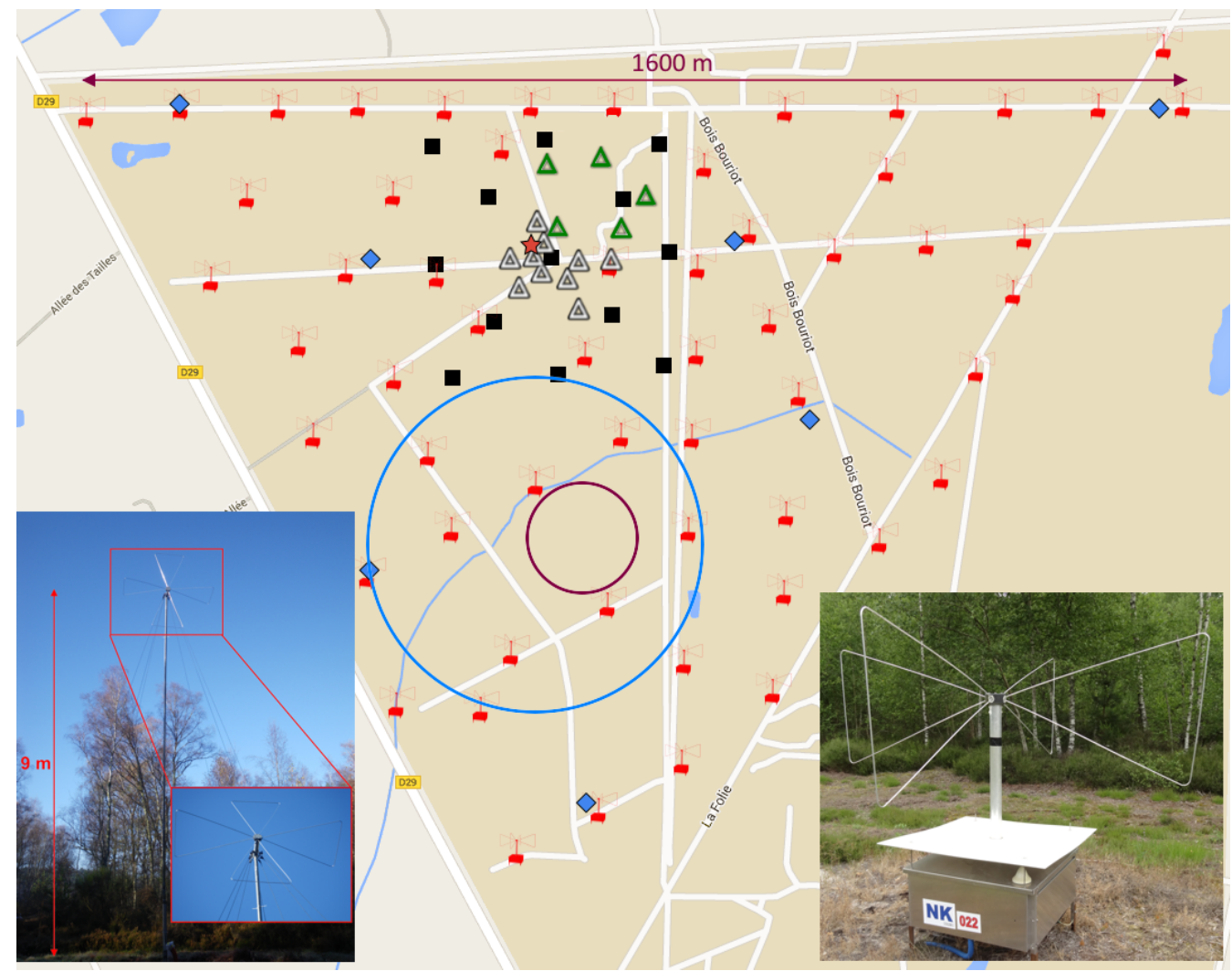

Figure 1. Map of the Nançay observatory (North on top). Red symbols: 57 CODALEMA autonomous radio detection stations; black squares: 13 scintillators; white and green triangles: 10 antennas of the compact array and 5 antenna cluster of the composite trigger; red star: "tripole" antenna; blue diamonds: 7 low-frequency antennas. Small purple circle: location of the FR606 LOFAR station; blue circle: extension of the NenuFAR radiotelescope. Bottom-right: a standalone detection station with the Butterfly antenna. The metallic box contains the electronics board crate in a shielded inner compartment. Bottom-left: a low frequency antennas on its post at $9 \mathrm{~m}$ height. 


\subsection{The Scintillator Detector Array (SD)}

The goal of the particle detector (notably described in [3]) is to provide the arrival direction, the size, the core location of the atmospheric shower and an estimation of the energy of the incident cosmic ray. It also provides a master trigger, which can be broadcasted to any other system throughout the observatory, among others the compact array of antennas and a dedicated station holding the same GPS module as the radio stations described later, for time-stamping. The particle detector array is made of 13 scintillators located on a grid with a variable spacing (approximately $120 \mathrm{~m}$ on the diagonal and $170 \mathrm{~m}$ on the NS and EW directions). It covers a $340 \times 340 \mathrm{~m}^{2}$ area whose center roughly corresponds to the former cabled radio array center of CODALEMA-2. The particle detector geometry has been designed such that its energy threshold is located at the knee energy, and the array reaches its maximum efficiency for an energy of $10^{16} \mathrm{eV}$, with a sensitivity level of 1 particle per $\mathrm{m}^{2}$.

\subsection{The standalone antenna array}

Since 2013, 57 standalone radio detection stations are installed over $1 \mathrm{~km}^{2}$, with an average spacing of about $150 \mathrm{~m}$ between stations. The latter feature 2 crossed-polarization "Butterfly" antennas (also used on AERA [4]), in the North-South (NS) and East-West (EW) directions (Fig. 1). Each of the EW and NS input channels is split in two equal parts by a $3 \mathrm{~dB}$ splitter. One part is amplified and filtered by a wide band analog, passive filter (in the $35-80 \mathrm{MHz}$ and the $150-200 \mathrm{MHz}$ bands). The filtered signal is compared to a voltage threshold, remotely adjustable. If the threshold is passed on one or both of the input channels, the level 1 (T1) triggering condition is fulfilled and the event is time-stamped by a GPS board. The daily T1 yield on a single station is about $10^{6}$. The other part of the signal (full band) continuously feeds a digitizer board, whose circular memory is frozen only when a T1 arises. A second triggering level (T2) is applied depending on the rise time of the signal [5], analyzed on-line by a local PC, and reducing the initial trigger rate by a factor of 1000 , therefore getting rid of the most prominent noise sources. A third triggering level (T3) can be applied at the array scale, selecting only interesting events having hit several stations in a given time window and constituting the air shower candidates. On average about 2 events per day are finally selected as cosmic ray events in time coincidence with the particle detector ones, above a threshold of $10^{17} \mathrm{eV}$. A very large bandwidth is still available, from $20 \mathrm{MHz}$ to $85 \mathrm{MHz}$ and from $110 \mathrm{MHz}$ to $200 \mathrm{MHz}$, thanks to the response of the Butterfly antenna and the fast digitizing system (1 GS/s). CODALEMA-3 is currently the only radio experiment able to access such a wide band at once.

The high frequencies could be of paramount interest to sample the Cerenkov ring. As shown in figure 2 from [6], close to the axis the expected Cerenkov signal in 120-200 MHz is much higher than in the band 30-80 MHz (with which it competes, giving a "doublepeak" structure), and furthermore its variation with the distance to the axis is faster. Unless a high antenna density is used (LOFAR case), it is difficult to correctly sample the electric field profile in $30-80 \mathrm{MHz}$, while using the information above $120 \mathrm{MHz}$ could greatly help determining the latter with only few antennas spaced by a few tens of meters (CODALEMA-3 case). This is currently studied on CODALEMA-3 events simulated with the SELFAS code [7].

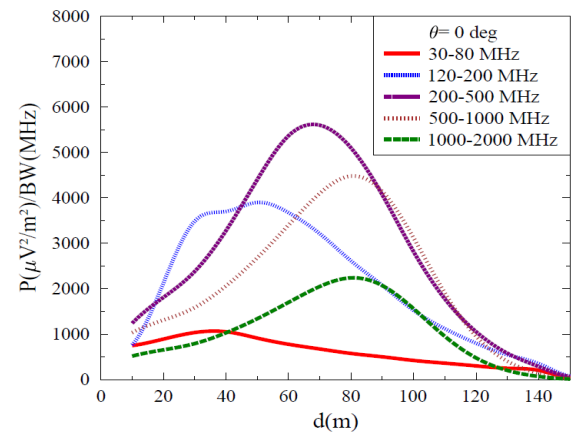

Figure 2. Electric field distribution vs distance to the shower axis for various frequency bands. Vertical proton at $10^{17} \mathrm{eV}[6]$. 


\subsection{A vertical component measurement}

A three-fold antenna system has been installed nearby the center of the particle detector to measure the complete electric field of air showers, i.e along 3 polarizations EW, NS and vertical.

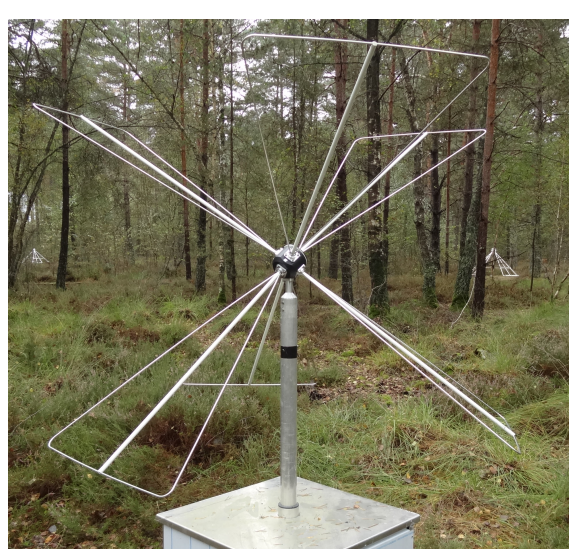

Figure 3. The tripole antenna. Indeed on all currently operating radio detection arrays, only the horizontal polarizations are measured, either directly along NS and EW directions or later projected on these axes. This allows reconstructing the vertical electric field component provided that the far-field assumption is valid, but although strong hints based on the theories of air shower radio emission tend to support this hypothesis (the main component should be the transverse current due to the separation of charges in the geomagnetic field), the latter has never been experimentally verified. Several attempts for measuring directly the vertical component of the E-field have been made but no convincing results have been shown, probably due to the low performances of the antennas used by now. We chose to reconsider the problem and to use 3 regular Butterfly antennas equipped with the LONAMOS LNA, rotated twice around the NS and vertical axes (Fig. 3). Triggered by the particle detector, the 3 signals are recorded with the same type of electronics as for the standalone stations. We have now to properly deconvolve it together with the regular autonomous station signals recorded at the same time, to derive conclusions about the initial hypothesis.

\subsection{A Compact Array as a composite triggering system}

Two majors problems occur with a sparse array of antennas or standalone stations in the band 30$80 \mathrm{MHz}$ : the detection range is quite small (few hundreds of meters) and the trigger selectivity is low in standalone mode. To study possible solutions to both these problems, a cluster of 10 antennas in dual linear horizontal polarisation has been installed, arranged in star-shape of $150 \mathrm{~m}$ width (24 to $146 \mathrm{~m}$ spacing). This cluster can operate in two observing modes:

- SD triggered, 20 channels recorded, $6 \mu$ s snapshots, 400 MHz ADC, bandwidth [10-200] MHz;

- self detecting mode (development in progress), with a continuous sampling of (up to) 8 channels, in circular polarisation (linear output added in quadrature), with an ADC at $100 \mathrm{MHz}$, a bandwidth of $50 \mathrm{MHz}$ ( $2^{\text {nd }}$ Nyquist band) and real-time software (using NVIDIA K20 GPU at 5 Tflops).

The SD triggered mode is used to test, off-line, the reconstruction algorithms intended to be implemented on-line in self-detecting mode. About $10^{3}$ radio events from $6.10^{5} \mathrm{SD}$ triggers have been reconstructed so far, allowing one to finely tune the reconstruction algorithms. Notably, the beamforming method helps avoiding triggering on natural and man made transient noise (i.e. lightnings, electric/electronic devices...) and moreover increases the sensitivity of antennas by a factor of $\sqrt{N}, N$ being the number of antennas in the cluster. We are currently developing an on-line detection scheme based on continuous sampling $\left(F_{S}=100 \mathrm{MHz}\right.$, bandwidth $\left.50 \mathrm{MHz}\right)$ of selected antenna output, stored in a ring buffer. A pre-conditioning is made by Fourier transforming each channel, synthesizing a circularly polarized channel, then computing the signal envelop for each successive time-window corresponding to the array time aperture $(500 \mathrm{~ns})$. It generates $\sim 2000$ beams on the sky $\left(\sim 2^{\circ}\right.$ apart) via 
beamforming, in which a search for beam(s) containing signal above some intensity threshold is conducted. Additional criteria (e.g. rise time, known RFI direction, polarization) may be used to increase the selectivity of the online trigger. This should allow deriving the size and the spacing between the antenna clusters needed to cover a very wide area in the case of a further giant observatory based on radio detection.

\subsection{EXTASIS and the low-frequency components}

The coherent emission due to the variation of the transverse current in the shower, also known as "geomagnetic effect", is expected to be the dominant effect in the global emission of the charged particles of the shower during its development in the atmosphere. A secondary process, related to the time-varying charge excess, has also been observed (see for example [8]). All the simulation codes show that the maximum of emission due to these mechanisms peaks at a frequency of $\sim 10 \mathrm{MHz}$, whatever the energy and the geometry of the shower are. Unfortunately, man-made broadcasting at low and medium frequencies ( 5 to $25 \mathrm{MHz}$ ) generate strong $\mathrm{AM}$ lines, that are visible all around the Earth. For that reason, all the recent experiments of radio detection of UHECR use a rather "clean" band located between AM and FM lines, i.e [30-80] MHz. Although several detections at low and very low frequencies have been made up to the 90's (see [9] and references therein) and have shown that the signal has a higher amplitude at low frequencies (below $10 \mathrm{MHz}$ ) than in the range [2080] $\mathrm{MHz}$, the various mechanisms proposed to explain this were not satisfactory. We therefore chose to reinvestigate low frequency measurements, by adding dedicated antennas in Nançay and by using the SELFAS simulation code to figure out what could be the emission mechanism. Especially, we will focus on the signal predicted by SELFAS. This new mechanism proposed in [9] is the coherent emission of the secondary electrons in excess in the shower front when reaching the ground level. These particles suffer a sudden deceleration when they disappear below the ground level; we proposed to call this the "sudden death mechanism", which creates the sudden death pulse (SDP). Detecting it is the goal of the EXTASIS project. 7 antennas on $9 \mathrm{~m}$ high poles have recently been installed within CODALEMA-3 (see figure 1). Their acquisition is triggered by the particle detector, and is made thanks to digital oscilloscopes (500 MS/s, $50 \mathrm{~ms}$ record, 8 bits). The very high frequency resolution allows us to clean the signal of the remaining transmitter lines in the frequency band of study (2$5 \mathrm{MHz}$ ). The antennas are classical Butterfly antennas with a modified LONAMOS LNA, adapted for the frequency range, with an East-West horizontal polarization and a vertical one, the SDP being expected to be mainly vertically polarized.

\section{Short overview of the main performances}

To illustrate the performances of the current experiments, we choose to present the signals of all CODALEMA-3/EXTASIS detectors for the same cosmic ray event in figure 4. From top to bottom and from left to right: time traces of the tripole antenna in 2 frequency bands; time traces of 1 lowfrequency antenna, in both polarizations; polarization figures of the Compact Array; map of the entire array, including the scintillator (thin circles) and the standalone antennas (bold circles). For the two latter, size of the circles is proportional to the amplitude of the signal, color scale represents the arrival times on individual detectors. The center plots are the power spectrum densities recorded on the standalone antennas, with a clear contribution on some of them up to $200 \mathrm{MHz}$. On the right of the figure, the time traces of the Compact Array antennas, and in the lower right corner the polarization figures for the 12 involved autonomous stations (polarization figures are shown without any antenna angular correction). 


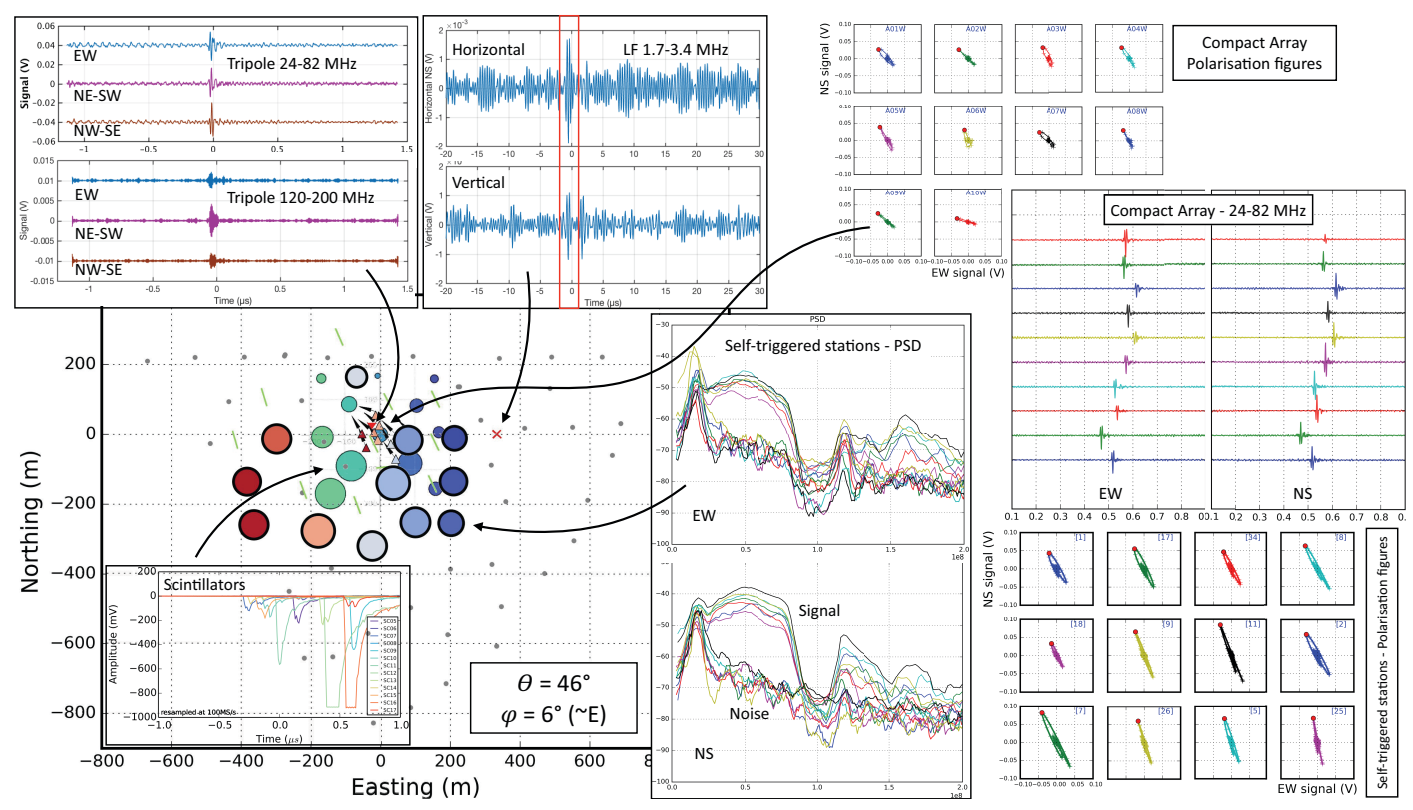

Figure 4. A hybrid event, seen by all the CODALEMA-3/EXTASIS arrays. See text for details.

\section{Conclusion}

The Nançay radio observatory now offers a variety of instruments dedicated to the radio detection of high energy cosmic rays. The main goal of this instrumental setup is to explore new or neglected ways of exploitation of the radio signal emitted by EAS, to fill the remaining gaps of the current detection method. Taking advantage of the very wide bandwidth of the CODALEMA-3 standalone station, we propose an alternative concept able to compensate for the large detector density currently necessary to reach a good sensitivity to the $X_{\max }$. The EXTASIS project aims at detecting the low frequency counterpart of the regular EAS radio signal, and the sudden death pulse expected to occur at the sudden deceleration of the air shower particles at ground. Last, thanks to a new designed tripole antenna, a study of the total electric field in [20-200] MHz will be performed, in order to verify the standard far-field hypothesis.

\section{References}

[1] J. Schulz, for the Pierre Auger collaboration, $34^{\text {th }}$ ICRC, The Hague, The Netherlands (2015).

[2] P. Schellart et al. (LOFAR Collaboration), AEA 560 A98 (2013).

[3] C. Rivière, PhD thesis, Université Joseph-Fourier - Grenoble I (2009). In french <tel-00526045>.

[4] The Pierre Auger Collaboration, JINST 7, P10011 (2012).

[5] D. Torres-Machado et al. (CODALEMA Coll.), 33 ${ }^{\text {rd }}$ ICRC, Rio de Janeiro, Brazil (2013).

[6] K. de Vries, O. Scholten and K. Werner, Astropart. Phys. 4523 (2013).

[7] F. Gate et al., 34 ${ }^{\text {th }}$ ICRC, The Hague, The Netherlands (2015).

[8] A. Bellétoile et al., Astropart. Phys. 69 50-60 (2015).

[9] B. Revenu et al., 33 ${ }^{\text {rd }}$ ICRC, Rio de Janeiro, Brazil (2013). 